\title{
L'EXPRESSION ÉMOTIONNELLE DANS LE DISCOURS POLITIQUE
}

\author{
Philippe Braud ${ }^{1}$
}

\begin{abstract}
Retraçant l'évolution de l'expression des émotions dans le temps, cet article montre que celles-ci sont désormais davantage assumées par les acteurs politiques et mieux étudiées par les chercheurs. On peut distinguer un petit nombre de registres émotionnels que les acteurs et partis mobilisent en fonction de leur positionnement dans le champ politique : il en va ainsi de l'activation de la peur ou du désir d'espérer, du recours au registre de la colère et de l'indignation, aussi bien qu'à celui de la fierté et de l'enthousiasme... Ces pratiques rhétoriques constituent des marqueurs identitaires.
\end{abstract}

L'appel explicite aux arguments d'ordre émotionnel dans la rhétorique politique a mauvaise presse, au moins dans les pays démocratiques. Les souvenirs sont encore si proches des désastreuses conséquences de la fanatisation des masses par des démagogues de droite comme de gauche. Les rhétoriques xénophobes du nationalisme ont provoqué l'enthousiasme d'une bonne partie de l'opinion en Allemagne, en Grande-Bretagne et en France, à la nouvelle du déclenchement de la première guerre ; elles ont facilité dans les colonies l'apologie des pires exactions tandis que les discours de haine de classes ont considérablement durci les affrontements entre patrons et syndicats,

1 Philippe Braud est Professeur émérite des universités à Sciences Po Paris.

Recherches en communication, $\mathrm{n}^{\circ} 41$ (2014). 
partis révolutionnaires et partis conservateurs voire simplement réformistes, tandis que les violences verbales échangées entre cléricaux et anticléricaux atteignaient leur paroxysme dans plusieurs pays d'Europe. L'impression, fondée, que ces appels appuyés à l'arrogance nationale, au mépris raciste et antisémite, à la haine religieuse ou à la haine de classe, détruisaient le lien social et contribuaient à paralyser le fonctionnement parlementaire et démocratique, a provoqué en retour, au lendemain de la seconde guerre mondiale, une durable stigmatisation du registre émotionnel dans le discours politique.

Néanmoins, cette attitude qui a longtemps influencé les problématiques des chercheurs en science politique, repose sur une forme d'aveuglement. Si les excès des démagogues sont la forme la plus visible, et la plus détestable, de la manipulation émotionnelle des peuples, cela ne signifie pas qu'ils constituent le mode exclusif de présence des affects dans la vie politique; et encore moins qu'une fois forclos ou marginalisés, ces excès laisseraient place à des comportements purement rationnels au cœur de la vie politique démocratique. Pour prendre conscience de l'irréductible présence des affects à tous les niveaux du débat et de la prise de décision politiques, il est nécessaire au préalable de lever les ambiguïtés de l'opposition émotionnel/rationnel. En effet, si l'on ré-éexamine les classiques distinctions webériennes de l'activité sociale 1) rationnelle en finalité, 2) rationnelle en valeur, 3) " affectuelle », 4) traditionnelle, on peut y lire la source de lourds malentendus (Weber, p. 55). Le registre émotionnel n'est nullement cantonné dans la troisième catégorie d'analyse, même si c'est ici que les motivations passionnelles sont les plus visibles. Cela est clair encore pour la seconde : quand les convictions déterminent seules les buts de l'activité sociale (c'est la définition de Weber), cela signifie que les références du sujet relèvent du souci de sa propre dignité et de l'estime de soi, que celle-ci soit associée, comme l'écrit Weber, au « sens du devoir » ou à la « quête de beauté » ou à la « grandeur d'une Cause ». Mais il faut aller plus loin. Si le comportement dit « traditionnel» n'est selon l'auteur, «très souvent qu'une manière morne de réagir à des excitations habituelles qui s'obstine dans la direction d'une attitude acquise autrefois » (Weber, p. 55), il n'en est pas moins utile d'y détecter une forme de "remise de soi », censée être sécurisante pour un acteur désireux d'éviter les coûts d'un examen plus approfondi de la justesse de ses motifs (d'agir ou ne pas agir). Enfin, dans la catégorie de « l'action rationnelle en finalité » Max Weber privilégie l'existence de «fins mûrement réfléchies ». En fait, lorsqu'il examine comment le sujet 
définit ses objectifs et identifie les moyens adéquats de les atteindre, il se place non plus dans une problématique rationnel $v / s$ émotionnel, mais plutôt dans une problématique contrôle conscient $v / s$ absence ou insuffisance de maîtrise. Pour lui, le sujet rationnel est ici avant tout le sujet conscient et maitre de soi ; ses ennemis sont l'ignorance de toutes les données utiles à son jugement ou la perte de contrôle de ses passions, et non pas le degré zéro de ses désirs. Cette approche de Weber rejoint d'ailleurs le sens commun le plus banal selon lequel un comportement « rationnel» se caractérise avant tout par la parfaite maîtrise de ses émotions, notamment celles qui sont les plus volatiles comme la peur et la haine, l'enthousiasme, l'admiration extatique ou le besoin d'illusions. Or, même dans cette hypothèse du contrôle, il serait erroné, et d'ailleurs illogique, d'en déduire l'absence totale d'émotions à l'œuvre dans la structuration des attitudes, des comportements et des opinions. S'il y a contrôle, c'est parce qu'il existe quelque chose à contrôler; et ces émotions « retenues » n'en sont pas moins opérantes, fût-ce sous des formes indécelables à une observation superficielle. Affirmer l'existence d'une instance purement rationnelle au sens où elle ignorerait toute interférence émotionnelle contredit d'ailleurs l'enseignement le plus récent des neurosciences sur le fonctionnement du cerveau humain, lequel ignore la distinction émotionnel/rationnel (Ledoux, 1997 ; Damasio, 1995).

Partant donc désormais du postulat de l'omniprésence de l'émotionnel dans le discours politique, on cherchera à évoquer d'abord ce qui se joue dans la simple construction d'une relation d'interlocution. On s'intéressera ensuite à la dimension, souvent plus visible, de l'appel aux émotions collectives par les acteurs politiques, avant de noter, enfin, le renouveau de l'expression de sentiments personnels au moins dans certaines conjonctures de communication.

\section{La charge émotionnelle inscrite dans l'interlocution}

La communication politique sous forme d'une prise de parole publique est intrinsèquement une forme d'affirmation de soi. La formule : « Je parle, donc j'existe» est vraie dans toute communication avec autrui, a fortiori dans la sphère du langage politique où la distinction entre la parole et l'action est spécialement problématique. En toute hypothèse, cette prise de parole a bien un contenu émotionnel minimal puisqu'elle joue avec des notions psychologiques comme l'exhibition d'une légitimité et d'une supériorité. En effet, toute expression par un 
politique d'une opinion, d'une analyse ou d'un plaidoyer, s'inscrit en général dans l'une ou l'autre des trois topiques les plus fréquemment décelables dans le champ politique :

- celle de l'expert. Le locuteur se place sur le terrain de celui qui détient un savoir spécialisé ou des informations privilégiées. «Je vais vous expliquer...» Que le locuteur soit stricto sensu un expert ou un responsable politique, en endossant ce type de langage, il ne se contente pas de développer une analyse explicite, il adopte aussi le métalangage de la supériorité savante, qui se dit dans le ton adopté, le recours à des arguments chiffrés, le langage sur-joué de la raison et de la logique, tous éléments qui le font paraître le plus proche du Vrai ;

- celle du représentant. Elle se fonde sur la légitimité issue des urnes et postule le droit de montrer le chemin à suivre pour protéger les acquis, affronter les menaces, identifier le cap à suivre dans l'action collective. Cette forme de supériorité l'autorise à parler au nom de ses électeurs, voire de l'ensemble des électeurs de sa circonscription quand ce n'est pas au nom du peuple tout entier, sans que puisse être vérifié l'acquiescement des représentés à chacune de ses affirmations. "Voilà ce que pensent ceux que je représente, voilà leurs aspirations, voilà ce qu'il faut entreprendre pour améliorer les choses...». Bien sûr ce mode de discours appelle des contestations et des rejets plus ou moins vigoureux de la part d'autres acteurs du champ politique, voire des représentés eux-mêmes. Mais si la politique est un univers de luttes permanentes, c'est bien parce que toute prise de parole d'un représentant contient une dose, infinitésimale ou majeure, d'agressivité à l'égard d'autres acteurs ;

- celle du défenseur des valeurs. La plupart des politiques aiment invoquer, pour justifier leurs choix, des convictions morales. Ils disent se battre pour l'Égalité, la Justice, la Solidarité, ou encore pour la Liberté, la Responsabilité individuelle, la Patrie, la République, le Bien commun, toutes catégories de références fortement chargées d'éthique. C'est, implicitement ou explicitement, chercher à se doter d'un statut moral. Le fait de dire et de répéter publiquement qu'ils combattent pour des valeurs, fonctionne, au moins à leurs yeux mais aussi aux yeux de ceux qui les approuvent, comme une sorte de ré-assertion permanente. Cette posture n'est évidement pas sans rapport avec la tentation de l'idéalisation de soi. Elle se voit renforcée par la pratique courante qui consiste, en période électorale surtout, à exhiber des motivations élevées à l'engagement : dévouement, sacrifice d'intérêts purement personnels, goût de l'action publique désintéressée. 
Toute affirmation de soi, a fortiori si elle est un tant soit peu virulente ou arrogante, a vocation à soulever une agressivité réactionnelle. Dans l'ordre du discours politique, c'est la règle générale. Aussi rencontret-on inévitablement des éléments rhétoriques qui visent à anticiper ou à gérer ces éléments de conflictualité. En direction des représentés au nom desquels (et à la place desquels) on s'exprime, les locuteurs éprouvent le besoin de réduire leurs défenses hostiles et d'activer au contraire leurs dispositions favorables. Cela implique de savoir « caresser» son public en flattant ses convictions, ses sentiments ou ses aspirations. Les locuteurs font aussi couramment état de leur proximité avec eux, dans le but évident de désarmer l'éventuelle impression de dépossession. Des affirmations récurrentes du type : «Je connais vos problèmes..., je vis les mêmes difficultés que vous... " constituent quelques-uns des éléments de langage les plus souvent utilisés dans ce but, avec les appels au «bon sens » ou aux « convictions » censés être le commun partage des élus et de leurs électeurs. A l'égard des rivaux ou des adversaires, une agressivité minimale est de rigueur. Elle s'exprime dans les tentatives de disqualification soit de ce qu'ils ont dit ou fait, soit de ce qu'ils sont. A cet égard les formules assassines, les épithètes condescendantes ou méprisantes sont souvent plus efficaces (au moins à court terme) que les argumentaires froids ou les contre-propositions soigneusement étayées ; pourvu cependant que le niveau de passion agressive qui s'y déploie demeure soigneusement millimétré afin d'en éviter les effets contre-productifs, ce qui dépend d'ailleurs beaucoup du type de bornes posées par la culture politique nationale et par les subcultures partisanes.

\section{L'appel aux émotions collectives}

C'est la manière la plus classique et la plus visible d'introduire le registre émotionnel dans le discours politique. En fait, cet appel a toujours constitué une ressource majeure des dirigeants et des autres acteurs politiques, quel que soit le régime dans lequel s'inscrivait leur action. Il est au principe même de ce que l'on appelle la politique symbolique, c'est-à-dire la mobilisation de fêtes, de cérémonials, de rituels, mais aussi la mise en place des catégories sémantiques de décryptage du passé, du présent et de l'avenir. Pour rester dans l'ordre proprement dit du discours politique, dont la fonction s'est trouvée démultipliée avec l'avènement de la démocratie politique et son avatar principal : les campagnes électorales, on peut relever, à des degrés 
d'intensité variable selon le niveau de conflictualité des consultations, l'inévitable instrumentalisation d'un certain nombre de dispositions émotionnelles des citoyens. En ce domaine Marion Ballet (2012) a conduit une enquête, exemplaire sur le plan méthodologique, portant sur les thèmes de campagne des candidats aux élections présidentielles françaises entre 1981 et 2007 (inclus). Son matériel empirique très riche inspirera fortement les développements qui suivent, tout en fournissant des illustrations quantifiées des thèmes abordés dans ce texte. On se contentera ici d'évoquer deux registres principaux : celui de la peur et de l'espoir, et deux autres plus spécifiques de certaines familles politiques : la fierté nationale et l'indignation contre l'injustice.

L'activation de la peur. De Gaulle avait lancé la formule célèbre : Moi ou le chaos. Sous des formes à peine différentes on notera qu'à sa suite tous les candidats importants, notamment les présidents sortants, ont brodé sur ce thème de la peur de l'instabilité, du désordre ou de la crise économique, et en ont fait un enjeu majeur du résultat de l'élection. Tantôt il s'agit de prendre en charge des peurs préexistantes, tantôt il s'agit de les dramatiser délibérément voire de les créer, pour mieux se donner le rôle de celui qui peut les exorciser. En d'autres termes, l'activation de la peur implique toujours le souci de rassurer, dans un jeu de stop and go très caractéristique de toute campagne électorale, de quelque famille que ce soit. La peur est toujours peur de quelque chose. Certains craignent pour leur épargne, d'autres pour leur emploi, d'autres et souvent les mêmes, pour leur sécurité, leur identité, l'avenir du pays ou celui de la planète... Selon leur appartenance politique, certains candidats sont disposés à dramatiser telles peurs plutôt que telles autres parce qu'ils s'estiment mieux placés pour ensuite rassurer. Le fait est qu'il existe entre partis et entre candidats un différentiel de crédibilité sur la capacité à apaiser les craintes, fondées ou non de la population, selon que les inquiétudes concernent l'emploi ou les hausses d'impôts, la gestion d'une crise financière ou le contrôle de l'immigration.

$\mathrm{Si}$, en raison des profits politiques qui en sont attendus, la dramatisation des peurs préexistantes est un élément constant de toute rhétorique électorale, et pas seulement aux extrêmes de l'échiquier politique, il n'en demeure pas moins qu'il s'agit d'une stratégie qui peut se révéler dangereuse, surtout pour les partis modérés. La peur en effet est une émotion très volatile, contagieuse, susceptible de s'autoalimenter au point de ne plus être maitrisable. Les paniques financières, les paniques de foules, la dénonciation virulente de boucs-émissaires, en constituent des exemples historiques évidents. Les formations à 
vocation gouvernementale se doivent donc d'être plus circonspectes dans l'appel aux peurs collectives tandis que les formations plus extrémistes y verront plus volontiers l'occasion de pêcher en eaux troubles.

L'activation du désir d'espérer. Le changement est le maître-mot de tous les programmes politiques même quand il est associé, ce qui est fréquent, au thème de la continuité ou au souci de «défendre les acquis ». Là encore, ce thème est décliné avec une intensité variable selon la position sur l'échiquier politique. Le paroxysme de l'espoir d'un avenir meilleur, sinon même enchanteur, aura été porté par les partis qui se proclamaient "révolutionnaires », un mot magique qui a galvanisé de nombreux mouvements politiques, à gauche surtout (révolution prolétarienne) mais aussi à droite (révolution fasciste, révolution nationale...). Si le thème est aujourd'hui en déclin marqué, il n'en demeure pas moins que les formations ou les candidats situés aux extrêmes sont les plus portés à formuler des promesses démagogiques, surtout si la possibilité d'être associés au pouvoir demeure lointaine ou exclue. Pour les partis de gouvernement au contraire, le risque est plus grand de provoquer par des promesses inconsidérées une déception proportionnelle à l'ampleur des illusions suscitées. Néanmoins, les conditions de la compétition électorale poussent ces candidats à un minimum de surenchère entre eux, surenchère dans laquelle les sortants affrontent un handicap spécifique puisque leurs adversaires pourront toujours leur reprocher de n'avoir pas été capables de satisfaire, quand ils étaient au pouvoir, les espoirs qu'ils prétendent désormais susciter. Ceci explique que les cycles d'alternance politique courts facilitent une certaine modération des promesses les plus inconsidérées. L'opposition qui aspire au pouvoir et qui l'a déjà exercé peu de temps auparavant, est davantage encline à la prudence dans ses promesses; à la fois parce que sa culture de gouvernement est encore forte en son sein, et parce qu'elle sait que son bilan passé pourra lui être rappelé. Au contraire, comme l'ont montré les premières décennies du débat politique sous la $V^{e}$ République, de trop longues alternances facilitent l'envol des promesses d'un avenir radieux et la mobilisation par l'opposition d'espérances démesurées.

Le registre de la colère et de l'indignation. L'indignation est l'expression d'une révulsion/stigmatisation, provoquée par le spectacle de ce qui est perçu comme intolérable. En ce sens elle est un rappel aux normes. L'émotion affichée peut être authentique ou simplement tactique parce que l'on présume sa capacité de rassemblement, au 
moins dans les couches de population que l'on cherche à séduire. C'est ainsi que l'indignation provoquée par « les cadeaux fiscaux » offerts aux patrons du CAC 40 est le fait de formations politiques déterminées, de même que, dans un autre secteur de l'échiquier politique, le sont les dénonciations $\mathrm{du}$ " péril islamiste ». De manière générale, l'appel à l'indignation de l'opinion est plus fréquemment le fait de partis situés aux extrêmes, comme le montrent les statistiques établies par Marion Ballet (2012, pp. 194-211, 242-260). Ce qui s'explique pour deux raisons : d'abord parce qu'ils se réclament de normes idéologiques (et morales) dissidentes, généralement plus rigides, par rapport à celles des autres formations politiques ; également parce que, minoritaires, ils ont intérêt à pratiquer une indignation bruyante pour tenter de se faire mieux entendre. Cependant l'indignation officielle de tous les partis est obligatoire devant les violences extrêmes, les injustices patentes, les scandales d'une exceptionnelle gravité, car l'opinion publique ne leur pardonnerait pas leur indifférence dès lors qu'elle elle-même serait profondément irritée. L'indignation réelle devant le spectacle de normes bafouées, engendre normalement la colère, laquelle peut constituer un levier, dangereux mais efficace, d'une mobilisation protestataire. En revanche, si elle est trop souvent sollicitée, l'indignation risque de tourner à vide et susciter beaucoup d'indifférence dans la majorité de l'opinion, tout en entretenant des foyers de mécontentement extrême dans des secteurs limités de la population.

Le registre de la fierté et de l'enthousiasme. Dans les démocraties contemporaines, y compris en France, la rhétorique des candidats aux élections se fonde de moins en moins sur ce registre. Tout se passe comme si avait été parfaitement intégré le caractère dévastateur ou illusoire des exaltations nationalistes du passé ou des ardeurs révolutionnaires. La fierté patriotique fait de moins en moins recette, même à l'extrême droite où elle est davantage remplacée par un sentiment catastrophiste du déclin. Quant à la fierté de travailler à construire un monde nouveau qui avait rendu si populaire le mot révolution non seulement à gauche chez les admirateurs de l'Union soviétique mais aussi à droite chez les sympathisants du fascisme et des régimes autoritaires, il s'agit d'une thématique devenue à peu près inaudible. La fierté se décline donc depuis un demi-siècle à un niveau beaucoup plus modeste. Fierté des sortants qui vantent leur bilan ; fierté de se dire « de gauche » par opposition aux « réactionnaires »; fierté encore, à droite, de s'afficher comme « responsable ». Fierté partout d'afficher des « valeurs », dont le contenu demeure le plus souvent fluide. 
En relation indirecte avec cette thématique de la fierté, on observera aussi le souci contemporain, qui s'exprime dans des législations, de bannir du débat politique les discours à caractère raciste, antisémite, islamophobe ou xénophobe, c'est-à-dire susceptibles de blesser la dignité d'un groupe de population. Nouvelle illustration de la volonté de rompre avec une culture politique du passé qui confondait l'expression de la fierté avec l'affirmation d'arrogance.

\section{L'évocation de sentiments personnels}

Cette dimension de la rhétorique émotionnelle dans les discours politiques n'est pas entièrement nouvelle même si elle semble connaître un regain de faveur depuis plusieurs décennies dans les campagnes électorales. Il convient en effet de distinguer deux formes différentes de cette stratégie discursive.

Les émotions personnelles prêtées aux citoyens. Depuis l'avènement du suffrage universel, les candidats en campagne ne se sont jamais privés de parler psychologiquement aux électeurs. On entend par là ces rhétoriques rituelles qui développent l'éloge du citoyen « raisonnable », « réfléchi », soucieux de s'informer, capable de discernement et de « responsabilité ». Ou encore, cet éloge moral du citoyen que l'on « sait » attaché à la justice, à la liberté, à l'égalité. Dans les partis de gouvernement, on a toujours cultivé les mises en garde contre un vote inspiré par «l'irritation » ou la « déception ». Une intéressante distinction de vocabulaire permettait (et permet encore) de prêter aux Français quelques défauts regrettables comme l'inconstance, l'indiscipline, le penchant pour des émotions excessives, tandis que les électeurs, eux, seraient toujours mus par cette sagesse qui leur ferait opérer, après mûre réflexion, le « meilleur choix », dicté par une conscience civique éclairée. Mais tous ces sentiments, avantageusement prêtés aux électeurs, gardaient quelque chose de convenu et surtout d'impersonnel. Il est vrai que, depuis l'avènement des partis de masses à la fin du XIX siècle, le débat politique a longtemps été dominé par les arguments d'ordre idéologique. Les affrontements tournaient autour de catégories collectives, fortement substantialisées : la grande ou la petite bourgeoisie, les classes moyennes, le prolétariat, toutes entités dont on louait le rôle historique ou stigmatisait leurs supposés noirs desseins. De même idéalisait-on, en tant que balises politiques, des icônes comme la Patrie, la République, la Nation, que d'autres vitupéraient avec acharnement. Cette structuration des intensités émotionnelles autour de concepts 
finalement abstraits laissait peu de place à un intérêt pour les sentiments « des gens », c'est-à-dire des personnes considérées indépendamment de leurs caractéristiques sociales ou partisanes, donc en dehors de leur appartenance à de vastes ensembles. Dès la Libération pourtant, on voit en France les orateurs communistes, notamment des femmes comme Jeannette Thorez-Vermeersch et les dirigeants de l'UFF (Union des Femmes Françaises), illustrer leurs discours politiques de scènes très personnalisées, empruntées à la vie quotidienne difficile des ouvriers, des chômeurs ou des mères de familles nombreuses. Par ailleurs, le porte-à-porte pratiqué par de nombreux candidats en campagne laissait la possibilité de s'entretenir des préoccupations et de l'état d'esprit des gens visités, fût-ce de façon superficielle. Avec le développement de la télévision et des nouvelles technologies de communication, un autre pas important est franchi. Il devient de plus en plus fréquent de voir insérés, dans les clips de campagne, des scènes de la vie quotidienne censées illustrer les inquiétudes des citoyens les plus ordinaires quant à leurs fins de mois ou à l'avenir scolaire de leurs enfants. Thèmes qui se sont invités dans les débats télévisés comme celui de Jacques Chirac « dialoguant» avec des jeunes lors de la campagne référendaire de 2005 ou celui du second tour de l'élection présidentielle, en $2007^{1}$.

Le message toujours sous-jacent mais devenu de plus en plus souvent explicite, est celui-ci : «Voyez, je vous comprends intimement et personnellement... ; vos joies et vos peines, ce sont aussi les miennes ». D'où ce langage quasi sentimental chez certains candidats qui culminera dans la campagne présidentielle de Nicolas Sarkozy et Ségolène Royal. Celle-ci, par exemple, s'adressant aux Français le 30 avril 2007, fait appel non seulement " à l'énergie » et " à l'audace » des Français (ce qui demeure classique) mais aussi, plus surprenant «à leur tendresse ». Mais déjà, certaines formulations de Valéry Giscard d'Estaing $^{2}$ ou de Jacques Chirac ${ }^{3}$ frisaient purement et simplement la déclaration d'amour. Ouvrant ainsi la voie à une nouvelle catégorie de rhétorique sentimentale.

1 Pour des exemples et des statistiques relatives à ce phénomène lors des élections présidentielles, voir Ballet, M. Op. cit. (p. 93 et sq). Selon elle, ce type d'occurrences a doublé dans le corpus qu'elle a étudié, entre 1988 et 2002, et augmenté encore de $50 \%$ entre 2002 et 2007 . Une croissance qui touche toutes les familles politiques.

2 Giscard d'Estaing, V. (1988) Le pouvoir et la vie, t. 1, chap. 6. Paris : Cie 12. : «A force de regarder les Françaises, je les ai vues et j'en suis tombé amoureux. Il faut prendre le mot dans le sens le plus précis ».

3 Jacques Chirac, Déclaration de candidature, 11 février 2002 : « Je connais et j’aime les Français... ». 
La mise en scène par les candidats de leurs émotions personnelles. Dans les campagnes électorales classiques des élections législatives $\mathrm{du} \mathrm{XX}^{\mathrm{e}}$ siècle, le phénomène se manifestait de façon extrêmement discrète. Quand le mode de scrutin était uninominal, les professions de foi des candidats distribuées dans les boites aux lettres, mentionnaient pratiquement toujours le statut matrimonial ainsi que, le cas échéant, l'existence d'enfants, voire de petits-enfants. Une manière implicite de se dire sensible aux joies de la vie de famille. Avec le temps, cette information s'est fréquemment enrichie de photos du couple ou/et de sa progéniture, voire des lieux habités (quand ils ne sont pas trop luxueux). La célèbre affiche de Giscard d'Estaing posant avec sa plus jeune fille (très discrètement cadrée, il est vrai) aura constitué un tournant significatif lors de la campagne présidentielle de 1974. Depuis cette époque, les entretiens accordés par des personnalités politiques à des journalistes de télévision ont de plus en plus souvent retenu le cadre d'une résidence familiale : le salon, la bibliothèque, le bureau, voire la cuisine, soigneusement cadrés bien sûr pour projeter l'image de quelqu'un d'humain, partageant les bonheurs simples de tout le monde (DSK préparant une omelette), ou suggérant la sérénité du sage au milieu de ses livres, l'harmonie du couple (photo du conjoint bien en évidence), l'affection pour des enfants voire.... l'attachement à un animal de compagnie (François Mitterrand ou Jacques Chirac avec leurs chiens). Mais depuis une quinzaine d'années, une nouvelle étape a été franchie. Les conseillers en communication poussent les candidats à la présidentielle à des épanchements personnels appuyés. Si certains renâclent (Lionel Jospin, François Hollande), d'autres jouent ce jeu avec dynamisme. En 2007, l'un (Nicolas Sarkozy) évoque ses origines étrangères et l'abandon de la famille par son père ; l'autre (Ségolène Royal) confie la trop grande sévérité de son père, la nécessité qui fut la sienne de se révolter contre lui pour s'accomplir. (Presque) tous les candidats à cette haute fonction de Président valorisent leur humanité, leur besoin de communiquer chaleureusement, leur plaisir à « rencontrer les gens ». Mais nul n'aura, à ce jour, été aussi explicite sur ce registre que Nicolas Sarkozy dans sa déclaration officielle de candidature, le 14 janvier 2007 : « Je pensais que la politique n'avait rien à voir avec mes émotions personnelles. J'imaginais qu'un homme fort se devait de dissimuler ses émotions (distanciation manifeste avec De Gaulle, Pompidou ou Mitterrand...). J'ai compris que l'humanité est une force et pas une faiblesse ». 


\section{Conclusion}

S'il ne faut pas surestimer la nouveauté du recours aux arguments d'ordre émotionnel dans le discours politique, le fait est néanmoins que, dans les démocraties européennes de l'après-guerre, et spécialement en France qui a été ici notre seul terrain d'observation, une évolution est clairement perceptible. Aux transes passionnelles de la vie politique de l'entre-deux guerres et de l'immédiat après-guerre, a succédé une profonde suspicion à l'égard des rhétoriques enflammées. Non pas au point de les éliminer mais certainement au point de les réguler (y compris juridiquement). En revanche, s'est dessinée une tendance nouvelle à personnaliser fortement le discours politique, notamment celui des candidats en campagne. Ce qui s'est traduit par une mise en scène croissante des émotions prêtées aux " gens », ainsi que par un étalage croissant de sensibilité chez les acteurs politiques. On peut se demander quelles sont les raisons de ce phénomène.

Un premier facteur explicatif tient au déclin marqué, dans le débat politique, des grandes narrations idéologiques qui noyaient les individus dans des masses (nation, classe, minorité ethnique ou religieuse) au profit d'une affirmation vigoureuse des personnes. Tout se passe comme si les électeurs se dégageaient de leurs gangues sociologiques et retrouvaient leur identité d'individus. Ce triomphe de l'individuation va de pair avec la psychologisation de la vie politique. Les citoyens auxquels s'adressent les politiques, ne sont pas seulement des agents sociaux ayant des intérêts collectifs à défendre, ils sont aussi des êtres humains habités par des émotions et des humeurs, qu'elles soient positives ou négatives.

Un second facteur explicatif, lié d'ailleurs en partie au précédent, se situe dans l'évolution des goûts du public en matière de rhétorique politique. Le style pompeux ou péremptoire, synonyme de supériorité, de distance mais aussi de médiocre sensibilité humaine, a perdu de son attractivité. Il convenait à une société où le fossé demeurait large entre ceux qui étaient " réputés savoir », et ceux qui devaient docilement se laisser instruire. Le développement rapide de couches sociales de mieux en mieux formées et éduquées a contribué fortement à cette évolution. La tendance à la réduction (apparente) des distances sociales favorise l'émergence d'un style politique plus décontracté, plus chaleureux, plus familier. Et du point de vue des candidats à la représentation politique, il a en outre l'avantage de suggérer la proximité du représentant avec ses concitoyens. 
Troisième facteur, enfin, l'apparition de nouvelles technologies de communication, depuis la généralisation de la télévision jusqu'à aujourd'hui, a permis le déploiement d'une communication de masse « chaude » au sens de MacLuhan, c'est-à-dire favorable à l'expression émotionnelle. Les vidéos peuvent montrer "les gens » dans leur quotidien, exprimant spontanément leurs craintes et leurs espérances. La communication sur Internet, les tweets et autres formes de messages à peu près affranchis de tout filtrage, favorise la réactivité immédiate dans l'expression des sentiments. Le personnel politique ne peut ni rester sourd à ces nouvelles informations, ni se passer d'utiliser ces nouvelles technologies. En ce sens, on peut dire que la politique, loin des carcans idéologiques de naguère, se rapproche des gens tels qu'ils sont.

\section{Références}

Ballet, M. (2012). Peur, espoir, compassion, indignation. L'appel aux émotions dans les campagnes présidentielles (1981-2007). Paris : Dalloz.

Boltanski, L. (1993). La souffrance à distance. Paris : Anne Marie Métailié.

Braud, P. (2007). Petit traité des émotions, sentiments et passions politiques. Paris : Armand Colin.

Charaudeau, P. (2005). Le discours politique. Les masques du pouvoir. Paris : Vuibert.

Damasio, A. (1995). L'erreur de Descartes, (trad.). Paris : Odile Jacob.

Edelman, M. (1977). Political Language. Words That Succeed and Politics That Fail. New York : Academic Press.

Giscard d'Estaing, V. (1988). Le pouvoir et la vie. Paris : Cie 12.

Gougou, F., Tiberj, V., Laplanche-Servigne, S., \& Peugny, C. (2007). Les mots des présidentielles. Paris : Presses de Sciences Po.

Ibarra, P., \& Kitsuse, J. (1993). Vernacular Constituents of Moral Discourse. Dans G. Miller \& J. Holstein (Éd.), Constructionist Controversies. Issues in Social Problems Theory (pp. 21-54). New York : Aldin de Gruyter.

Lamizet, B., \& Tétu, J.-F. (Éd.). (2004). Emotions dans les médias. Mots. Les langages du politique, 75 .

Le Bart, C. (1998). Le discours politique. Paris : PUF.

Ledoux, J. (1997). The Emotional Brain. The Mysterious Underpinnings of Emotional Life ( $2^{\mathrm{e}}$ éd.). New York : Schuster.

Lutz, C., \& Abu-Lughod, L. (Éd.). (1990). Language and the Politics of Emotion. Cambridge : Cambridge University Press.

Marcus, G. (2008). Le citoyen sentimental. Emotions et politique en démocratie (trad.) Paris : Presses de Sciences Po.

Neumann, R., Marcus, G., \& Crigler, A. (Éd.). (2007). The Affect Effect. Dynamics of Emotion in Political Thinking and Behavior. Chicago : University of Chicago Press. Weber, M. Economie et société, tome I, chap. 1, § 2, (trad.). Paris : Plon Agora. 